University of South Carolina

Scholar Commons

$9-2000$

\title{
Regulation of SUP Expression Identifies Multiple Regulators Involved in Arabidopsis Floral Meristem Development
}

\author{
Hajime Sakai \\ Beth A. Krizek \\ University of South Carolina - Columbia, krizek@sc.edu \\ Steven E. Jacobsen \\ Elliot M. Meyerowitz
}

Follow this and additional works at: https://scholarcommons.sc.edu/biol_facpub

Part of the Biology Commons

\footnotetext{
Publication Info

Published in The Plant Cell, Volume 12, Issue 9, 2000, pages 1607-1618.

(c) The Plant Cell 2000, American Society of Plant Physiologists.
}

This Article is brought to you by the Biological Sciences, Department of at Scholar Commons. It has been accepted for inclusion in Faculty Publications by an authorized administrator of Scholar Commons. For more information, please contact digres@mailbox.sc.edu. 


\title{
Regulation of SUP Expression Identifies Multiple Regulators Involved in Arabidopsis Floral Meristem Development
}

\author{
Hajime Sakai, ${ }^{1,4}$ Beth A. Krizek, ${ }^{2}$ Steven E. Jacobsen, ${ }^{3}$ and Elliot M. Meyerowitz \\ Division of Biology, 156-29, California Institute of Technology, Pasadena, California 91125
}

\begin{abstract}
During the course of flower development, floral homeotic genes are expressed in defined concentric regions of floral meristems called whorls. The SUPERMAN (SUP, also called FLO10) gene, which encodes a C2H2-type zinc finger protein, is involved in maintenance of the stamen/carpel whorl boundary (the boundary between whorl 3 and whorl 4) in Arabidopsis. Here, we show that the regulation of SUP expression in floral meristems is complex, consisting of two distinct phases, initiation and maintenance. The floral meristem identity gene LEAFY (LFY) plays a role in the initiation phase through at least two pathways, which differ from each other in the involvement of two homeotic genes, APETALA3 (AP3) and PISTILLATA (PI). AP3, PI, and another homeotic gene, AGAMOUS (AG), are further required for SUP expression in the later maintenance phase. Aside from these genes, there are other as yet unidentified genes that control both the temporal and spatial patterns of SUP expression in whorl 3 floral meristems. SUP appears to act transiently, probably functioning to trigger a genetic circuit that creates the correct position of the whorl $3 /$ whorl 4 boundary.
\end{abstract}

\section{INTRODUCTION}

Flowers of angiosperms develop from floral meristems, small groups of undifferentiated cells derived from shoot apical meristems. One of the characteristics that often separate floral meristems from shoot meristems is the pattern of organ initiation. In Arabidopsis, shoot apical meristems form organs in a spiral pattern, whereas floral meristems form organs in a whorled pattern. Four types of floral organs are produced in four concentric whorls: sepals in the outermost whorl (whorl 1), petals in whorl 2, stamens in whorl 3, and carpels in the innermost whorl (whorl 4) (Smyth et al., 1990). Boundaries of these whorls are established very early in flower development, as evidenced by the distinct expression pattern of the homeotic genes APETALA3 (AP3), PISTILLATA (PI), and AGAMOUS (AG) in whorl-specific patterns (Yanofsky et al., 1990; Jack et al., 1992; Goto and Meyerowitz, 1994). The initial expression of these genes is detected in early stage 3 flowers formed after 3 days of floral meristem development. Accordingly, the pattern of whorls (the prepattern of ho-

\footnotetext{
${ }^{1}$ Current address: DuPont AG Genomics, Delaware Technology Park 200, 1 Innovation Way, Newark, DE 19714-6104.

${ }^{2}$ Current address: Department of Biological Sciences, University of South Carolina, Columbia, SC 29208.

${ }^{3}$ Current address: Department of MCD Biology, UCLA, P.O. Box 951606, Los Angeles, CA 90095-1606.

${ }^{4}$ To whom correspondence should be addressed. E-mail Hajime. Sakai@usa.dupont.com; fax 302-631-2607.
}

meotic gene expression) has to be set before this stage. Several genes are involved in whorl patterning. Floral meristem identity genes appear to be partly responsible for the establishment of these boundaries. Loss of function of the $L E A F Y$ ( $L F Y$ ) gene, for instance, changes the floral whorl structure to a partially spiral pattern (Huala and Sussex, 1992; Weigel et al., 1992). The Ify phenotype is synergistically enhanced by mutations in APETALA1 (AP1), another floral meristem identity gene. In the double mutant ap1 lfy, the whorl pattern and other floral meristem characteristics are severely disrupted (Weigel et al., 1992). A similar but less severe phenotype is observed in flowers lacking the function of the UNUSUAL FLORAL ORGANS (UFO) gene, which is required for the proper identity of floral meristems (Ingram et al., 1995; Levin and Meyerowitz, 1995; Wilkinson and Haughn, 1995).

Several mutations are known to alter the expression domains of the floral homeotic genes. Recessive mutations in the SUPERMAN (SUP) gene specifically affect the boundary between whorls 3 and 4 (Schultz et al., 1991; Bowman et al., 1992). In the sup mutant, this boundary is shifted toward the center of the floral meristem, resulting in ectopic expression of $A P 3$ and $P I$ in the whorl 4 region and the formation of extra stamens at the expense of carpels (Bowman et al., 1992). This alteration of the whorl $3 / 4$ boundary in the sup mutant occurs progressively from stage 4 onward, after the initial expression of $A P 3$ and $P I$ has been established (Sakai et al., 1995). From these observations, SUP was thought to be a factor in maintaining this boundary after the whorl prepattern had been established. 
The cloning of SUP has enabled us to study the function of this gene at the molecular level. We report here studies of SUP expression and genetic interactions between SUP and other floral regulatory genes, which reveal the presence of multiple genetic pathways that control the patterning of floral whorl boundaries. Our results show that SUP expression is regulated by floral meristem identity genes as well as by floral organ identity genes. By analyzing SUP expression in different floral mutants and in transgenic plants that ectopically express homeotic genes, we found that one or more additional, as yet unidentified whorl-specific factors act to define SUP expression in the inner part of whorl 3. Further, genetic analyses of double mutants revealed that only a transient period of SUP expression is required for setting the right pattern of the whorl $3 / 4$ boundary in stage 4 and older floral meristems. This indicates that SUP functions as a trigger of balanced whorl proliferation that is later inherited without further SUP RNA input.

\section{RESULTS}

\section{Genetic Interaction between sup and the Floral Meristem Identity Mutants}

The floral meristem identity genes $L F Y$ and $A P 1$ are involved in early flower development, partly establishing the whorl pattern (Irish and Sussex, 1990; Huala and Sussex, 1992; Mandel et al., 1992; Weigel et al., 1992; Bowman et al., 1993). To examine a possible interaction between these genes and SUP, we analyzed double mutants of sup with Ify as well as of sup with ap1. In the strong Ify mutant (Ify-6), floral organs exhibit sepal/carpel-like characteristics with various degrees of mosaic features. The number of organs in Ify flowers was reduced, and the whorled floral structure was partially converted to a spiral pattern. The phenotype of the flowers of the double mutant sup-1 lfy- 6 was very similar to
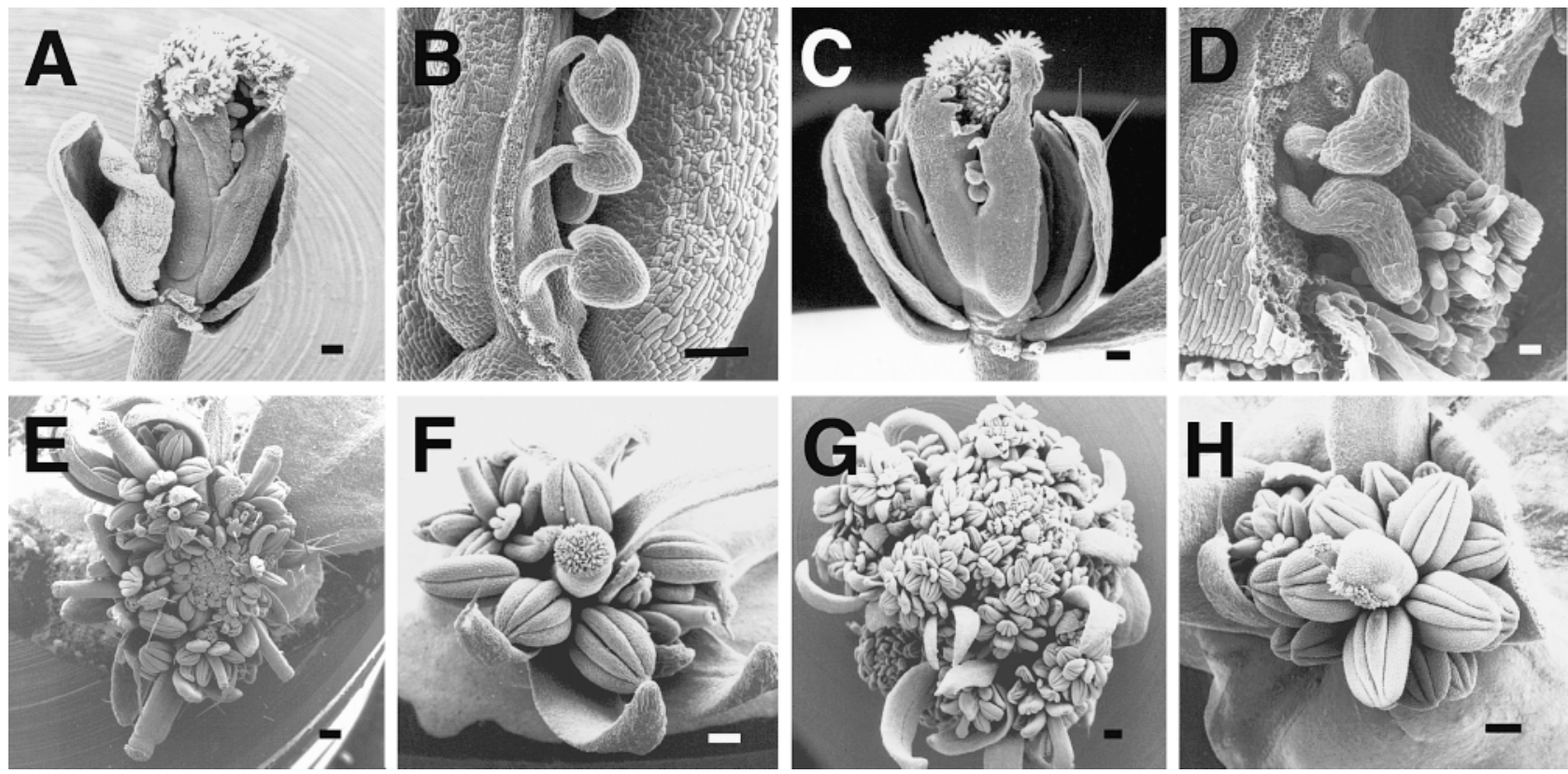

Figure 1. Scanning Electron Microscopy of sup Ify and sup ap1 Double Mutants.

(A) Mature Ify-6 flower. Two sepal-like organs were removed to show the organs formed inside.

(B) Ovules of a Ify- 6 flower, with the amphitropous configuraton seen in wild type.

(C) Mature sup-1 Ify-6 flower. Several sepal-like organs were removed to show the organs formed inside.

(D) Ovules of a sup-1 Ify-6 flower. They exhibit an elongated structure seen in sup mutants (Gaiser et al., 1995).

(E) ap 1-1 inflorescence.

(F) ap 1-1 flower. Extra flowers are formed in the axils of the first whorl organs.

(G) sup-1 ap 1-1 inflorescence. Most of the floral organs formed on the inflorescence are stamens.

(H) sup-1 ap 1-1 flower. Stamens are formed interior to whorl 2. A very reduced carpelloid structure with some staminoid characteristics is formed in the center of the flower, similar to the structure found in sup-1 flowers (Bowman et al., 1992).

Bars in $(\mathbf{A})$ to $(\mathbf{C})$ and $(\mathbf{E})$ to $(\mathbf{H})=100 \mu \mathrm{m}$; bar in $\mathbf{( D )}=10 \mu \mathrm{m}$. 
Table 1. Organ Number of Ify- 6 and sup-1 Ify- 6 Flowers ${ }^{a}$

\begin{tabular}{lllll}
\hline & $\begin{array}{l}\text { Leaf- or } \\
\text { Sepal-like } \\
\text { Organs }^{b}\end{array}$ & $\begin{array}{l}\text { Carpels or } \\
\text { Sepal/Carpel } \\
\text { Mosaic Organs }\end{array}$ & $\begin{array}{l}\text { Total No. } \\
\text { Organs }^{c}\end{array}$ & \\
\hline Ify-6 & $9.6 \pm 1.5$ & $4.7 \pm 1.1$ & $14.4 \pm 1.3$ & $n=30$ \\
sup-1 lfy-6 & $9.7 \pm 2.6$ & $4.4 \pm 1.3$ & $14.1 \pm 2.9$ & $n=37$ \\
\hline
\end{tabular}

a Numbers given are the mean \pm SD.

${ }^{b}$ The leaf-like or sepal-like class includes organs that are mainly leaflike but had some petaloid or staminoid character.

${ }^{c}$ Flowers 5 and 14 were analyzed for organ numbers. $n$, number of flowers counted.

that of the flowers of Ify- 6 (Figures $1 \mathrm{~A}$ and $1 \mathrm{C}$ ). The same number and types of organs were formed in sup- 1 Ify- 6 and Ify-6 flowers (Table 1), demonstrating that Ify is epistatic to sup. However, ovules produced in the double mutant were often elongated, like those seen in sup mutants (Gaiser et al., 1995), indicating an additive phenotype with regard to ovule development (see Figures 1B and 1D).

Another gene that controls the establishment of floral meristem identity is $A P 1$. The strong loss-of-function mutation ap 1-1 shows transformation of the two outer whorls into inflorescence-like structures, often forming leaves with axillary flowers in whorl 1 and no petals (Figures 1E and 1F). Whorls 3 and 4 are not affected by the mutation. The double mutant sup-1 ap1-1 exhibited phenotypes characteristic of both single mutants; whorls 1 and 2 were ap1-like, and whorls 3 and 4 were sup-like (Figures $1 \mathrm{G}$ and $1 \mathrm{H}$ ). The number of stamens formed in the double mutant flower was the same as the number formed in the sup mutant flower (Table 2). The genetic interaction between sup and ap1 thus appears to be additive.

\section{SUP Expression in Floral Meristem Identity Mutants}

To further characterize the interaction between SUP and the floral meristem identity genes, we examined SUP expression in these mutants. SUP expression is detected from late stage 3/early stage 4 in the inner part of the ring of floral meristem that will later give rise to stamen primordia (Figures 2A and 2B; Sakai et al., 1995). By stage 8, when stamen primordia are completely separated from the adjacent carpel primordia, SUP expression is no longer detectable. In stage 9 flowers, SUP expression is detected in developing ovule primordia. This expression later becomes restricted to the stalks of the ovules, called funiculi. We refer to this expression as late SUP expression and to that in floral meristems as early SUP expression.

In the strong Ify- 6 mutant, early SUP expression was not detected in floral meristems at any stage (Figures $3 \mathrm{H}$ and 3I). However, later SUP expression was seen in ovules that occasionally formed in Ify flowers (data not shown). This re- sult is in agreement with the phenotype of the double mutant sup Ify, which produces flowers with a floral whorl structure indistinguishable from lfy single mutants but produces ovules that are sup-like (Figures 1A to 1D).

Consistent with the phenotypes observed in double mutants with sup (Figures $1 \mathrm{E}$ to $1 \mathrm{H}$; Levin and Meyerowitz, 1995), the strong loss-of-function mutants of two other genes controlling floral meristem identity (ap1-1 and ufo-2) cause no alteration of early SUP expression (data not shown).

\section{SUP Expression in Floral Homeotic Mutants}

The expression of the homeotic genes $A P 3, P I$, and $A G$ precedes early SUP expression by approximately half a day. This observation raises the possibility that these floral homeotic genes act upstream of SUP and regulate SUP expression in whorl 3 (Sakai et al., 1995). To explore this, we analyzed SUP expression in ap3, pi, and ag mutant flowers. To be as quantitative as possible, all floral tissues were fixed and processed in the same way, and the mutant and wildtype sections were placed on the same slides. The tissues on the slides were then hybridized with the ${ }^{35}$ S-labeled SUP antisense probe.

In strong ap3-3 and pi-1 mutants with nonsense mutations (Jack et al., 1992; Goto and Meyerowitz, 1994), decreased early SUP expression was detectable in late stage 3 /early stage 4 flowers (Figures $2 \mathrm{C}$ and $2 \mathrm{E}$ ). The spatial expression pattern, however, was not affected in these young mutant flowers. At stage 5 and later, SUP expression was further reduced, to an undetectable level (Figures 2D and $2 \mathrm{~F})$. In contrast to the early expression, late SUP expression in funiculi of ovules was not affected by these mutations (data not shown). In the strong ag-3 mutant, no reduction of the initial SUP expression in stage 4 flowers was observed; however, no SUP expression was detected in whorl 3 primordia at stage 5 and older (Figures $2 \mathrm{G}$ and $2 \mathrm{H}$ ). SUP was subsequently and repeatedly expressed in the stage 4 floral meristems that formed in the interior region of the indeterminate ag flowers. These results show that the class $B$ homeotic genes $A P 3$ and $P I$ positively regulate early SUP expression from its initial stage, whereas the class $C$ gene

Table 2. Stamen and Axillary Flower Numbers in ap1-1, sup-1, and sup-1 ap 1-1 Flowersa

\begin{tabular}{lllcl}
\hline Genotype & Stamens & & \multicolumn{2}{c}{ Axillary Flowers $^{\mathrm{b}}$} \\
\hline ap1-1 & $\mathrm{ND}^{\mathrm{c}}$ & & $1.8 \pm 0.7$ & $n=20$ \\
sup-1 & $11.3 \pm 1.7$ & $n=25$ & $0 \pm 0$ & $n=25$ \\
sup-1 ap1-1 & $12.4 \pm 1.6$ & $n=25$ & $2.2 \pm 1.0$ & $n=20$ \\
\hline
\end{tabular}

a Numbers given are the mean $\pm \mathrm{SD} ; n$, number of flowers counted. ${ }^{b}$ Flowers 5 and 14 were analyzed for organ numbers.

${ }^{\mathrm{C} N D}$, not determined. 


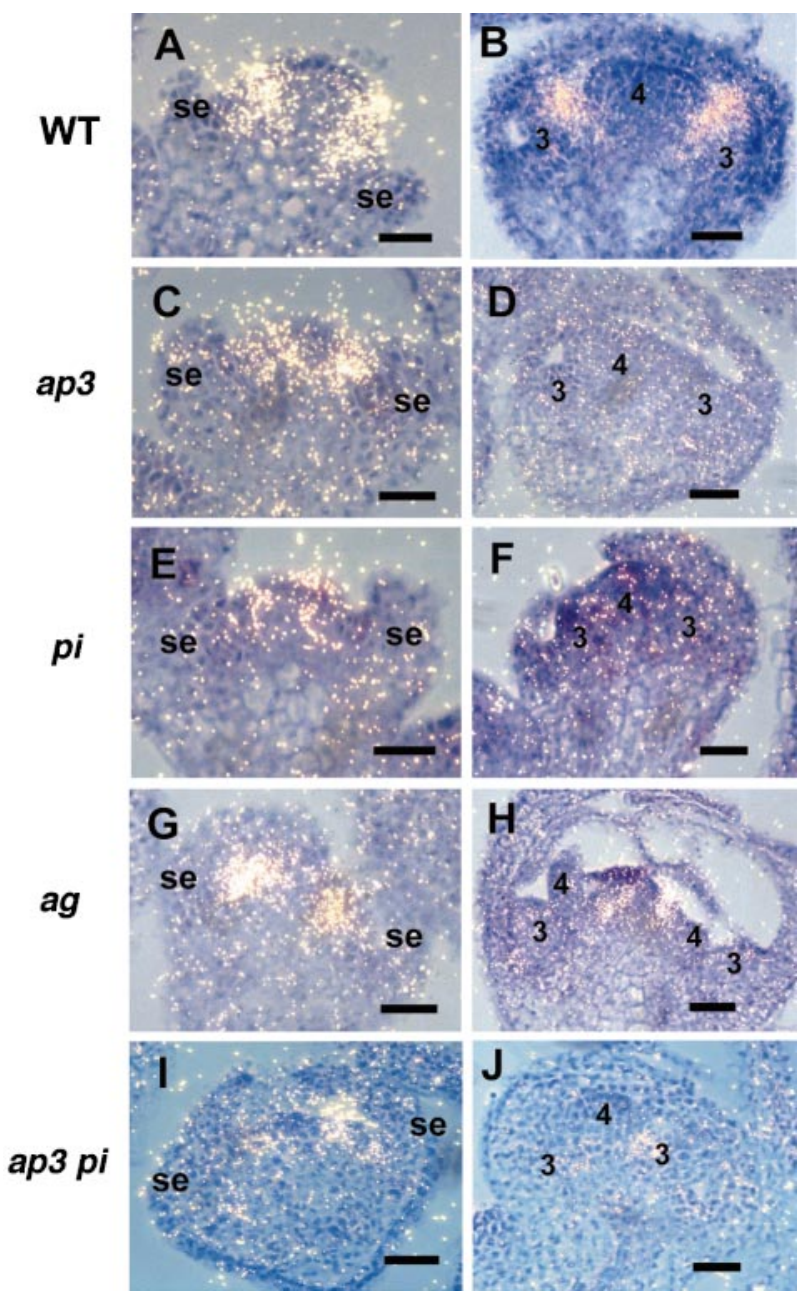

Figure 2. Expression of SUP RNA in Wild-Type (Landsberg erecta), ap3, pi, ag, and ap3 pi Mutant Flowers.

(A) and (B) Wild type (WT).

(C) and (D) ap3-3.

(E) and (F) pi-1.

(G) and (H) ag-3.

(I) and (J) ap3-3 pi-1.

SUP expression is shown in late stage 3/stage 4 in (A), (C), (E), (G), and (I) and in stage 5/6 in (B), (D), (F), (H), and (J). Flowers were sectioned longitudinally. In situ hybridization with the SUP antisense probe was performed as described by Sakai et al. (1995). To detect weakly expressed SUP RNA, sections were exposed on film for 2 months after hybridization. se, sepal primordium; 3 , whorl 3 primordium; 4, whorl 4 primordium. Bars $=10 \mu \mathrm{m}$.

$A G$ is involved only in maintenance of SUP expression from stage 5 onward.

We further examined SUP expression in the class A floral homeotic mutant ap2-2, which is a strong allele with a splice junction alteration that acts to truncate the encoded protein. In ap2-2, SUP was expressed in the area of the floral mer- istem corresponding to the third whorl (data not shown). Later, SUP was ectopically expressed in the carpelloid organs formed in whorl 1 of the ap2-2 mutant flower, which correlated with late SUP expression in ovule primordia. These results show that the class $A$ homeotic gene $A P 2$ is not required for the normal regulation of early SUP expression.

\section{Strong Class B Mutations Are Not Epistatic to sup}

Although the loss of $A P 3$ and $P I$ function reduced early SUP expression, SUP RNA was still detectable in stage 4 floral meristems (Figures $2 \mathrm{C}$ and $2 \mathrm{E}$ ). To investigate whether this residual expression retains any function, we examined double mutants between sup-1 and the strong class B mutants in detail. Previously, flowers of the double mutants had been shown to exhibit a phenotype very similar to those of ap3 and pi single mutants (Schultz et al., 1991; Bowman et al., 1992). Our detailed analysis, however, revealed that several features of double mutant flowers were distinctly different from single mutant flowers. In strong ap3-3 and pi-1 mutants, filamentous organ structures frequently formed in whorl 3 (Figures $4 \mathrm{~A}$ and $4 \mathrm{H}$ ). The organs occasionally showed carpelloid characteristics and were sometimes fused to the central gynoecium in whorl 4. The formation of these structures was reduced acropetally such that these filamentous organs were more frequently found in early-forming flowers compared with late-forming flowers (Table 3). The late-forming flowers of ap3-3 and pi-1 often had no solitary organs in whorl 3 but instead formed a central gynoecium that consisted of several unfused carpels. In the double mutants sup-1 ap3-3 and sup-1 pi-1, no formation of the filamentous organs in whorl 3 was observed (Table 3 and Figures 4D and 4K). Moreover, flowers of the double mutants produced only one gynoecium interior to whorl 2 sepals, and there was no obvious acropetal difference in floral organ formation. The gynoecium of the double mutants was more uniformly fused than that of the ap3-3 and pi-1 single mutants (Figures $4 \mathrm{C}, 4 \mathrm{~F}$, and $4 \mathrm{G}$ ). When developing flowers were examined by scanning electron microscopy, the gynoecium of the double mutants appeared to have originated from six fused organ primordia-two lateral and four medial-a pattern that resembled the ontogeny of the wild-type whorl 3 organs (Figure 4F). Formation of any other organ primordia distinct from the central gynoecium was not observed inside the gynoecium at or near stage 7 (Figure 4G), whereas organ primordia were formed from two whorls in ap3-3 and pi-1 and displayed some irregularity in the numbers and their fusions (Figure 4C; Jack et al., 1992).

To further investigate the developmental changes of double mutant flowers, we analyzed histological features of sup-1 pi-1 and sup-1 ap3-3. As early as stage 6, when the carpel primordia were formed from the floral meristem, we observed a difference in the development of the central region. In the single mutants pi-1 and ap3-3, the center of the floral meristem developed into carpel primordia (Figures $5 \mathrm{~A}$ to 
35S::AP3 35S::PI

35S::AP3

35S::PI 35S::AG
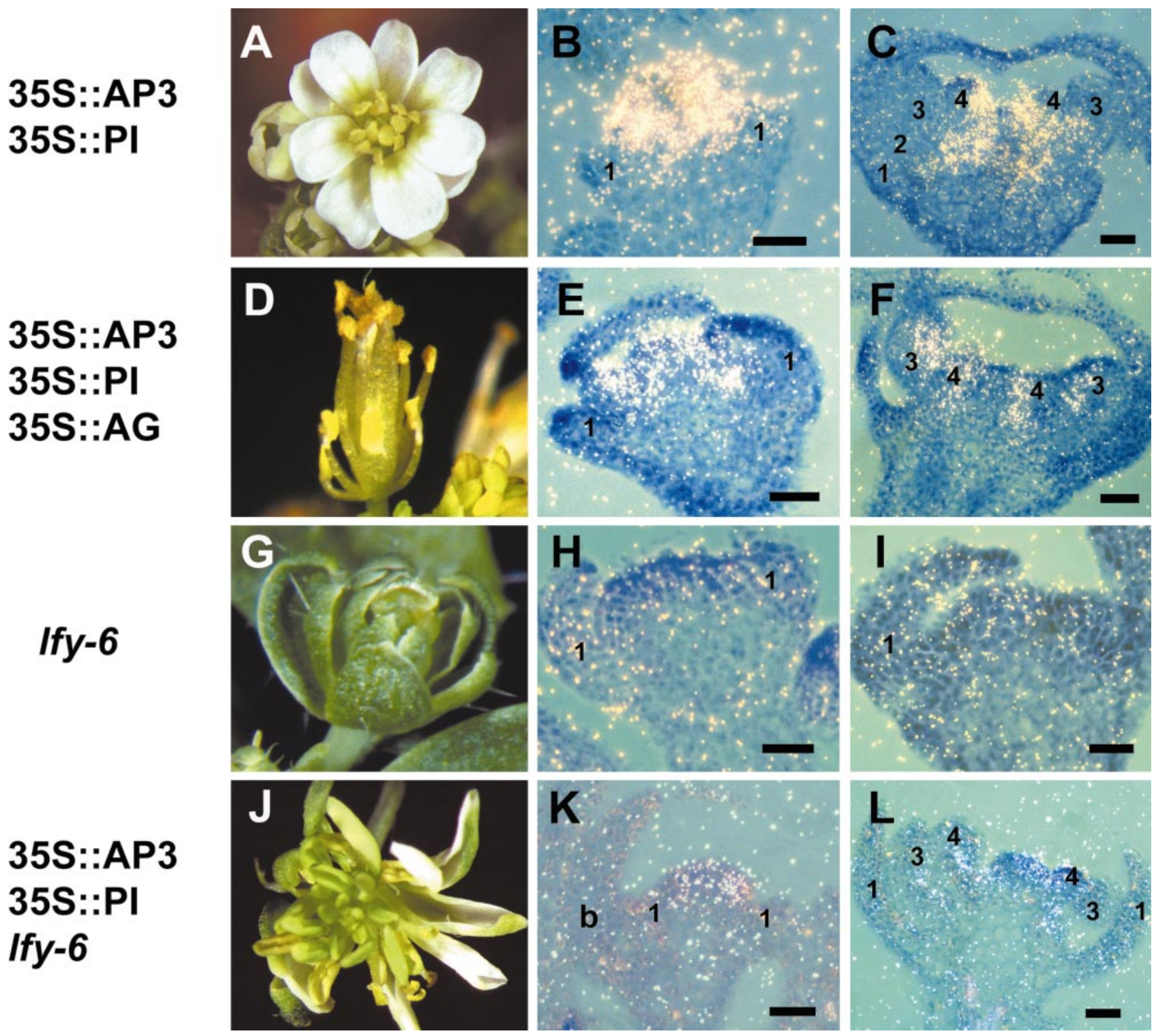

Figure 3. Expression of SUP RNA in Flowers of the Ify Mutant and Transgenic Plants.

Flowers were sectioned longitudinally.

(A) to (C) 35S::AP3 35S::PI transgenic plants. Flowers ectopically expressing AP3 and PI have transformation of sepals to petals in whorl 1 and carpels to stamens in whorl 4 with indeterminate characteristics (Krizek and Meyerowitz, 1996). (A) A mature flower; (B) Stage 4 flower; (C) a stage 6 flower.

(D) to (F) 35S::AP3 35S::PI 35S::AG transgenic plants. All floral organs are transformed to stamens or staminoid organs in the triple transgenic lines expressing $A P 3, P I$, and $A G$ ectopically. (D) A mature flower; (E) a stage 4 flower; (F) a stage 6 flower.

(G) to (I) Floral organs of the strong Ify- 6 mutant carrying a nonsense mutation are mostly sepal- or carpel-like. (H) A stage 4 flower; (I) a stage 5 flower.

(J) to (L) 35S::AP3 35S::PI Ify-6 transgenic plants. Ectopic expression of AP3 and PI partially rescues the organ identity defect of Ify-6, producing more stamen- and petal-like organs (Krizek and Meyerowitz, 1996). (J) A mature flower; (K) a stage 4 flower; (L) a stage 6 flower.

1, whorl 1 primordium; 2, whorl 2 primordium; 3 , whorl 3 primordium; 4, whorl 4 primordium; b, bract. Bars $=10 \mu \mathrm{m}$. 

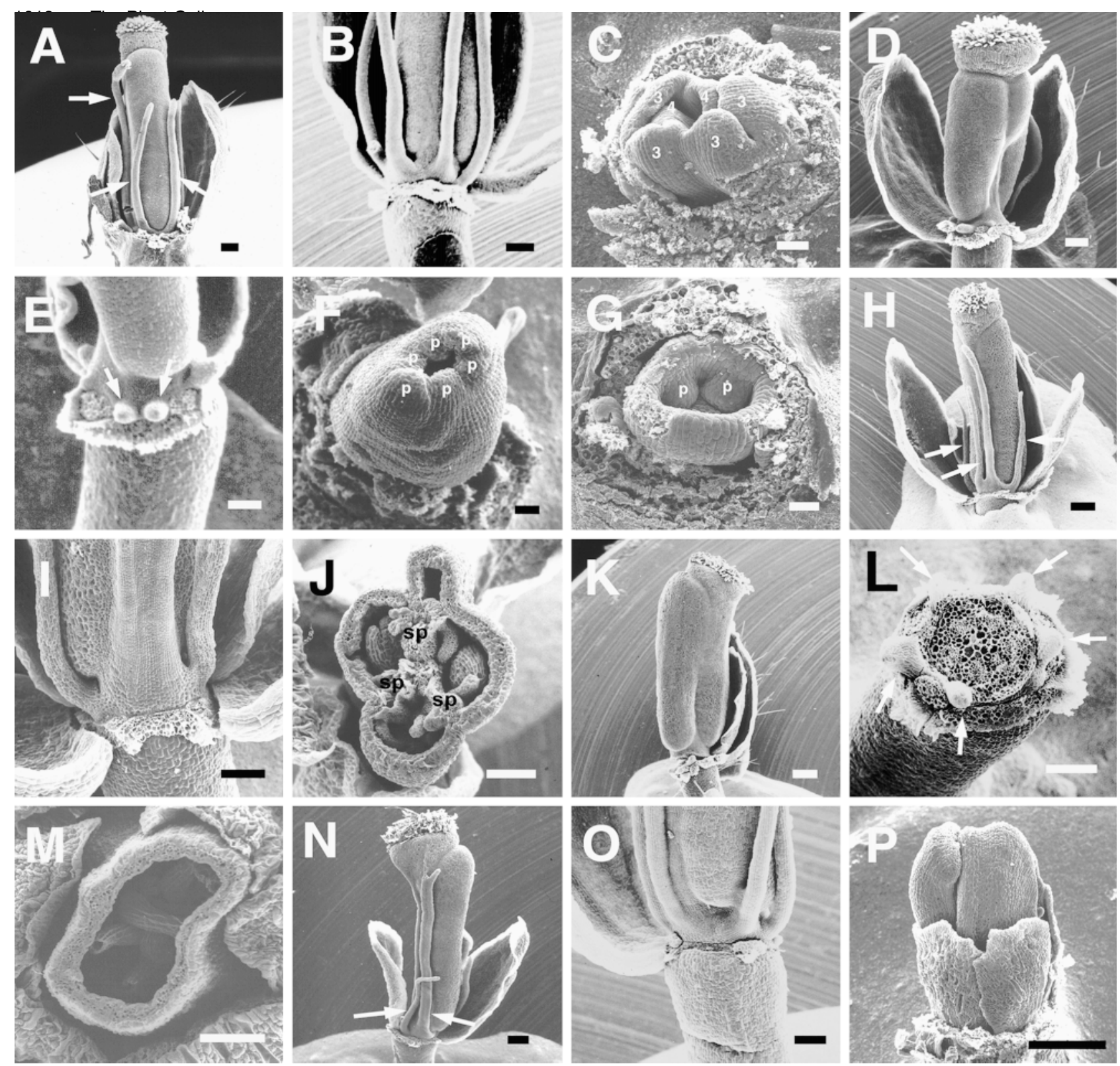

Figure 4. Scanning Electron Microscopy of the Double Mutants sup ap3, sup pi, and ap3 pi.

(A) to (C) ap3-3 flowers. (A) Mature flower with solitary filamentous organs in the third whorl (indicated by arrows). Several sepals in whorl 1 and 2 were dissected. (B) The receptacle region of a flower. Mature nectaries are not formed at the base of the third whorl organs. (C) Young flower in stage 7. Organ primordia are formed in whorl 3 (3) as well as in whorl 4 (4). All sepal primordia were removed from the flower bud.

(D) to (G) sup-1 ap3-3 flowers. (D) Mature flower with no filamentous organs formed between the central gynoecium and the sepals in whorls 1 and 2. (E) The receptacle region. Nectaries with a mature appearance are formed at the base of the central gynoecium (indicated by arrows). (F) Young stage 7 flower with the central gynoecium developed as six fused lobes in the distal region, indicating that it consists of six organ primordia (p). All sepal primordia were dissected from the flower. (G) Young stage 7 flower with the central gynoecium formed as a fused structure of several primordia $(p)$ from a very early developmental stage. All sepal primordia were removed.

(H) to (J) pi-1 flowers. (H) Mature flower with solitary, filamentous organs in whorl 3 (indicated by arrows). Several sepals in whorls 1 and 2 were removed. (I) The receptacle region of a flower. Mature nectaries were not formed at the base of the third whorl organs. (J) Transverse section through a mature pi-1 gynoecium. sp, septa between carpels.

(K) to (M) sup-1 pi-1 flowers. (K) Mature flower with several sepals in whorls 1 and 2 dissected. No organs were formed between the central gynoecium and the sepals in whorls 1 and 2. The surface of the gynoecium does not show any clear septum along its whole length. (L) The receptacle region of a mature flower. All distal floral organs were removed to show the nectaries (indicated by arrows), which look well developed. (M) Transverse section of a mature gynoecium. Note that the septum is not evident within the central gynoecium.

(N) to (P) ap3-3 pi-1 flowers. (N) Mature flower showing solitary or fused filamentous organs formed between whorls 2 and 4 (indicated by arrows), similar to the organs seen in the single mutants ap3-3 and $p i-1$. (O) The receptacle region of a mature flower with immature nectaries. (P) Young stage 7 flower. The carpel primordia develop as separate organs, as seen in ap3-3 and pi-1.

Bars in (C), (F), and (G) $=10 \mu \mathrm{m}$; all other bars $=100 \mu \mathrm{m}$. 
$5 \mathrm{C}, 5 \mathrm{~J}$, and $5 \mathrm{~K}$ ), whereas in sup-1 ap3-3 and sup-1 pi-1, the region remained as an undeveloped meristematic structure (Figures $5 \mathrm{D}$ to $5 \mathrm{~F}, 5 \mathrm{M}$, and $5 \mathrm{~N}$ ). This structure was further detected through the carpel development of the double mutants. A similar meristematic structure has also been observed in sup single mutants (Schultz et al., 1991; Bowman et al., 1992; Sakai et al., 1995). For sup pi double mutants, similar ontogeny has been noted in sup-2 (flo10) pi-1 double mutants (Schultz et al., 1991). The phenotypic correlation between sup and the double mutants is observed in the further floral organ development. In sup-1 ap3-3 and sup-1 pi-1, the base of the inner gynoecium surface often formed several bulges, which later developed into placental structures with ovules (Figures $5 \mathrm{~F}$ and $5 \mathrm{~N}$ ). Occasionally, the outgrowth of the inner surface was so extensive that a new gynoecium structure was later formed inside of the original gynoecium (Figure 50). Such overproliferation of the placental region was not detected in the single mutants ap3-3 and pi-1 (Figures 5C and 5K). Although the bulged structure was often congenitally fused with the gynoecium, it resembled the formation of extra whorls of stamen primordia in sup mutants, which also differentiated after the development of organ primordia in whorl 3 . The subsequent gynoecium development of the double mutants also differed from single mutants and wild type. In double mutants, the placental region was restricted to the bulged structure, which did not extend along the entire length of carpels (data not shown). In accordance with the irregular placentation, a complete septum did not form in the gynoecium of double mutants, and often no clear evidence of the region between the valves on the outer epidermis of gynoecium was detected (Figures $4 \mathrm{~K}$ and $5 \mathrm{I})$.

Flowers of the double mutants further differed from the ap3 and pi single mutant flowers in the formation of nectaries. In wild-type flowers, nectaries form at the outer bases of stamen filaments. In ap3-3 and pi-1, nectaries rarely developed, and those that did usually appeared small and immature (Figures $4 \mathrm{~B}$ and $4 \mathrm{I})$. On the other hand, in double mutants, nectaries morphologically indistinguishable from those of wild type frequently formed at the base of the central gynoecium (Figures 4E and 4L). Although the ontogenetic linkage of nectaries to whorl 3 is not proven, the formation of well-developed nectaries suggests that the sup ap3 and sup pi double mutants have restored the structure of the whorl 3 region, which is reduced in ap3 or pi mutants.

These phenotypes strongly indicate that the gynoecium of the double mutants forms from whorl 3 organ primordia and that the development of the whorl 4 organs is arrested even in the background of pi and ap3 loss of function. Accordingly, these strong class B mutations are not epistatic to sup. Rather, they exhibit an additive phenotype with the sup mutation.

\section{Loss of Both AP3 and PI Functions Does Not Completely Eliminate SUP Expression}

To investigate whether the residual expression of SUP in ap3-3 or pi-1 single mutants depends on the activity of the partner gene ( $P I$ or $A P 3$, respectively), we examined SUP expression in the ap3-3 pi-1 double mutant. $F_{2}$ double mutant plants were identified by polymerase chain reaction (PCR)-based genotyping (see Methods). For comparison, sections of the wild type and the pi-1 and ap3-3 single mutants were placed on the same slides with sections from the ap3-3 pi-1 double mutant and processed simultaneously. SUP expression in whorl 3 was weakly detected in the double mutant with an intensity similar to that observed in the single mutants (Figures $2 \mathrm{l}$ and $2 \mathrm{~J}$ ). This further shows that an additional gene or genes aside from AP3 and $P I$ are involved in induction of SUP expression at the initial phase.

The phenotype of ap3-3 pi-1 also demonstrates that SUP has a function even in the absence of both $A P 3$ and $P I$ activity. The isolated ap3-3 pi-1 double mutant plants exhibited a phenotype indistinguishable from the single mutant ap3-3 and pi-1 plants (Figures $4 \mathrm{~N}$ to $4 \mathrm{P}$ ). In the ap3-3 pi-1 flowers, filamentous organs were often produced in whorl 3 , like those produced in the single mutants. The development of nectaries

Table 3. Number of Organs between Whorl 2 and the Central Gynoecium in ap3, pi, sup ap3, and sup pi Flowers

\begin{tabular}{lllllllll}
\hline & \multicolumn{7}{l}{ No. of Filamentous Organs in Whorl ${ }^{\mathrm{b}}$ (Solitary Organ No./Fused Organ No.) } \\
\cline { 2 - 7 } Floral Order ${ }^{\mathrm{a}}$ & ap3-3 & & sup-1 ap3-3 & pi-1 & \\
\hline $1-4$ & $2.7 \pm 1.9 / 1.0 \pm 1.1$ & $n=44$ & $0 \pm 0 / 0 \pm 0$ & $n=51$ & $2.0 \pm 1.8 / 1.2 \pm 1.3$ & $n=33$ & $0 \pm 0 / 0 \pm 0$ & $n=36$ \\
$5-8$ & $1.0 \pm 1.3 / 0.7 \pm 1.0$ & $n=42$ & $0 \pm 0 / 0 \pm 0$ & $n=60$ & $0.6 \pm 1.0 / 0.6 \pm 1.0$ & $n=36$ & $0 \pm 0 / 0 \pm 0$ & $n=36$ \\
$9-12$ & $0.5 \pm 0.9 / 0.3 \pm 0.5$ & $n=44$ & $0 \pm 0 / 0 \pm 0$ & $n=60$ & $0.1 \pm 0.2 / 0.3 \pm 0.8$ & $n=36$ & $0 \pm 0 / 0 \pm 0$ & $n=36$ \\
$13-16$ & $0.2 \pm 0.5 / 0.4 \pm 0.6$ & $n=44$ & $0 \pm 0 / 0 \pm 0$ & $n=60$ & $0.2 \pm 0.6 / 0.2 \pm 0.6$ & $n=36$ & $0 \pm 0 / 0 \pm 0$ & $n=36$ \\
$17-20$ & $0.1 \pm 0.4 / 0.1 \pm 0.3$ & $n=44$ & $0 \pm 0 / 0 \pm 0$ & $n=60$ & $0.1 \pm 0.5 / 0.2 \pm 0.5$ & $n=36$ & $0 \pm 0 / 0 \pm 0$ & $n=36$ \\
\hline
\end{tabular}

${ }^{a}$ Four flowers were grouped together for organ counting according to their ages, that is, floral formation along the inflorescence (the earliest produced flower is counted as 1 of the floral order).

${ }^{b}$ Numbers given are the mean $\pm \mathrm{SD} ; n$, number of flowers counted.

c Solitary organs were counted separately from organs that were fused to the central gynoecium along more than half of their length. 

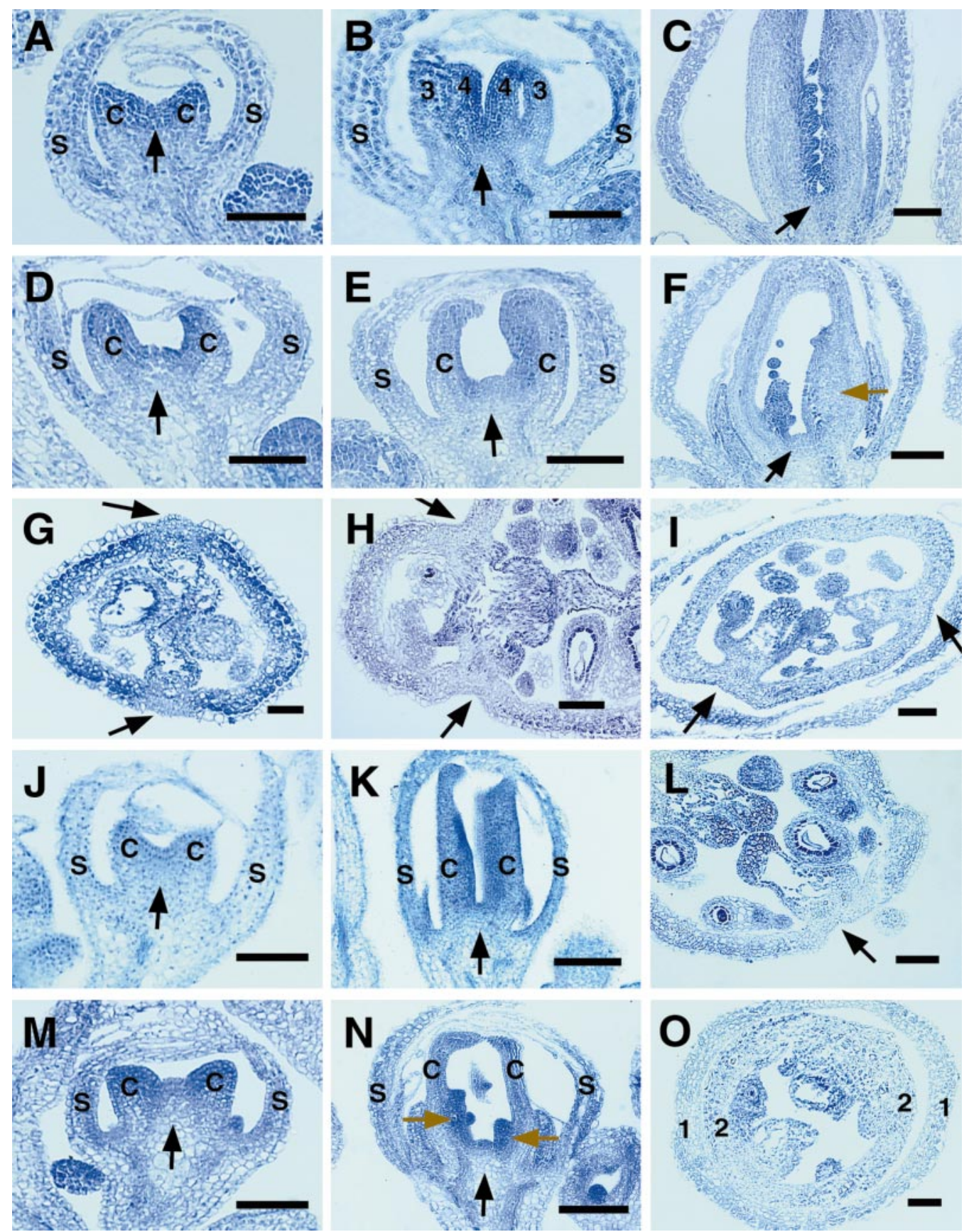

Figure 5. Histological Analyses of ap3-3, pi-1, and the Double Mutants sup-1 ap3-3 and sup-1 pi-1.

(A) to (C) and (H) ap3-3.

(D) to (F) and (I) sup-1 ap3-3.

(G) Wild type (Landsberg erecta).

(J) to (L) pi-1.

(M) to (O) sup-1 pi-1.

(A), (D), (J), and (M) Developing flowers at stage 6; (B) and (E) developing flowers at stage 7; (C) and (F) young flowers at stage 9; (K) and (N) young flowers at stage 8; and (G) to (I), (L), and (O), transverse sections of gynoecium. The central region (indicated with black arrows) shows an undeveloped meristematic structure in double mutants. Brown arrows on $\mathbf{( F )}$ and $(\mathbf{N})$ show the region formed inside of the carpel primordia. Arrows on (G) to (I) and (L) indicate the valval area of gynoecia. In wild type (G), the valval area forms a distinct tissue structure with small epidermal cells. Similar structures are seen in ap3-3 (H) and pi-1 (L) gynoecia but are not obvious in double mutants (I). Occasionally, a gynoecium (designated 2) is formed within a gynoecium (designated 1 ) in double mutants (0).

C, carpel primordium; S, sepal; 3 , whorl 3 organ primordium; 4 , whorl 4 organ primordium. Bars $=50 \mu \mathrm{m}$. 
was also impaired, similar to what was observed in ap3-3 and pi-1 single mutants (Figure 4O). Given how this phenotype differs from that of sup-1 ap3-3 or sup-1 pi-1, SUP appears to have a function, even in the ap3-3 pi-1 double mutant.

\section{$A P 3, P I$, and $A G$ Are Not Sufficient to Activate SUP Expression in Whorls 1 and 2}

Our expression analysis showed that the homeotic genes specifying stamen identity, $A P 3, P I$, and $A G$, positively regulate SUP expression in the adaxial part of whorl 3 . To determine whether these homeotic genes were sufficient to induce SUP expression, we further examined SUP expression in flowers constitutively expressing these homeotic genes. Transgenic plants constitutively expressing both $A P 3$ and $P I$ under the $35 \mathrm{~S}$ promoter of cauliflower mosaic virus form petals in whorls 1 and 2 and stamens in all interior whorls (Krizek and Meyerowitz, 1996). The initial SUP expression in these flowers was found in a pattern very similar to that seen in wild type but at a higher level (Figures 3B and 3C). Later, SUP expression was detected in the stamen primordia that formed interior to whorl 3. This expression pattern was very similar to that seen in the 35S::AP3 transgenic plants (Sakai et al., 1995). Although SUP expression was observed in the stamen primordia formed interior to whorl 3, it was not detected in the central region of the floral meristem, where all three of the $A P 3, P I$, and $A G$ genes were expressed. This indicates that another factor is present that prevents SUP expression in the center of whorl 4.

SUP was expressed in a similar pattern in flowers that ectopically expressed all three of these homeotic genes (AP3, $P$, and $A G$ ) under the control of the constitutive $35 \mathrm{~S}$ promoter from cauliflower mosaic virus. These transgenic plants produced flowers with stamens and staminoid organs in all floral whorls (Figure 3D). In the flowers of these transgenic plants, SUP was expressed in the whorls interior to whorl 3 but not in the outer two whorls and not in the very center of the floral meristem (Figures 3E and 3F). The expression pattern was indistinguishable from that seen in the 35S::AP3 35S::PI double transgenic flowers. Similar results were obtained in experiments using two different transgenic plants: $35 \mathrm{~S}: \mathrm{AG}$, constitutively expressing $A G$ under the $35 \mathrm{~S}$ promoter, and pAP3::AG, expressing $A G$ in whorl 2 under the $A P 3$ promoter (data not shown). These results demonstrate that $A P 3, P I$, and $A G$ are not sufficient to induce SUP expression in whorls 1, 2, and the center of floral meristems - a further indication that another factor is restricting SUP expression to whorl 3.

\section{LFY Regulates SUP through Two Pathways}

$L F Y$ positively regulates the class $\mathrm{B}$ homeotic genes $A P 3$ and $P I$ (Weigel and Meyerowitz, 1993). To elucidate possible interactions between $L F Y$ and $A P 3 / P I$ in SUP regulation, we analyzed SUP expression in the Ify mutant with constitutive
A

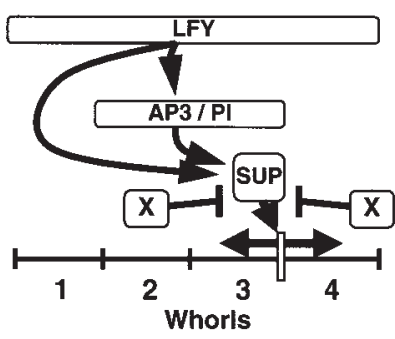

B

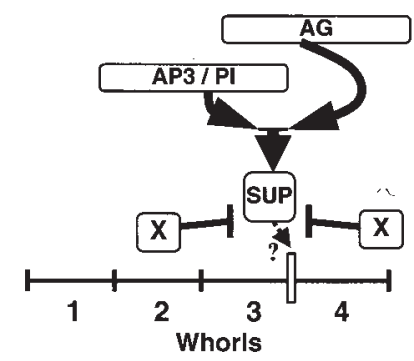

Figure 6. Schematic Presentation of SUP Gene Regulation.

The regulation of early SUP expression has two distinct phases, the initiation phase (stage 3/4) (A) and the maintenance phase (stage 5 and later) (B). In the first phase, SUP is controlled through at least three pathways; two pathways are regulated by $L F Y$ (one mediated through $A P 3 / P I$ ), and another pathway restricts the spatial and temporal pattern, which is regulated by one or more as yet unidentified genes $(X)$. The role of $X$ can also be filled by an activator specific for whorl 3. SUP expression at this phase is sufficient to fulfill the function of maintaining the whorl $3 / 4$ boundary. In the maintenance phase, AP3, PI, and AG control SUP expression. The activity of each homeotic gene is absolutely required for the maintenance of SUP expression. The SUP function in this phase is as yet undetermined.

AP3 and PI expression (35S::AP3 35S::PI Ify-6). Flowers of these plants formed organs with more staminoid characteristics than seen in Ify- 6 (Figure 3J; Krizek and Meyerowitz, 1996). In this background, SUP expression was detected in the whorl 3 region of floral meristems (Figures $3 \mathrm{~K}$ and $3 \mathrm{~L}$ ), although this expression was substantially lower than in the 35S::AP3 35S::PI plants. This result shows that early SUP expression can be partially recovered through activation of $A P 3$ and $P I$ in the absence of $L F Y$ function; further, at least two pathways of SUP regulation are controlled by $L F Y$, one mediated through $A P 3$ and $P I$, and the other mediated not through these class $B$ genes but in some other way. Given that SUP is expressed in whorl 3 from stage $3 / 4$ in this transgenic Ify mutant background, as was seen in wild type, the whorl-specific factor that restricts early SUP expression temporally and spatially appears not to be affected by $L F Y$ activity. 


\section{DISCUSSION}

\section{Role of $L F Y$ in SUP Expression}

Our results show that the floral meristem identity gene $L F Y$ regulates SUP expression in floral meristems. The double mutant between sup-1 and the strong nonsense mutant allele Ify- 6 is indistinguishable from lfy- 6 with regard to whorl structure and floral organ numbers. However, the double mutant produces ovules similar to those of sup-1. This phenotype is in agreement with the SUP expression pattern; that is, early SUP expression but not late expression is repressed by the loss of $L F Y$ activity. Previously, the class $\mathrm{B}$ homeotic genes were shown to be partly regulated by $L F Y$ (Weigel and Meyerowitz, 1993). Examination of SUP expression in Ify- 6 transgenic plants ectopically expressing $A P 3$ and $P I$ showed partial recovery of SUP early expression. This demonstrates that $L F Y$ regulates early SUP expression via two pathways, one of which is mediated through $A P 3 / P I$ (see Figure $6 \mathrm{~A}$ ). The full requirement of $L F Y$ for SUP early expression is rather remarkable, considering that the loss of $L F Y$ function alone does not fully eliminate the floral whorl structure. This may reflect that the genetic circuit establishing the whorl structure is not the same as the one maintaining it. Recently, $L F Y$ was shown to act on downstream genes through direct binding to target sites (Parcy et al., 1998; Busch et al., 1999). The 6.7-kb-long SUP genomic fragment that complements the sup mutation also contains three sites matching the consensus sequence for LFY binding (Sakai et al., 1995; H. Sakai, unpublished data). Future analyses of the SUP promoter will determine whether the non-AP3/PI LFY pathway is mediated by direct LFY binding.

\section{Floral Homeotic Genes AP3, PI, and AG Activate Early SUP Expression in Two Phases}

By analyzing the expression pattern in corresponding mutants, we showed that the floral homeotic genes AP3, PI, and $A G$ are positive regulators of SUP, although their requirement differs temporally. Early SUP expression is substantially lessened and becomes undetectable by stage 5 in the loss-of-function mutants of $A P 3$ and PI. Thus, these class $B$ homeotic genes are partly required for the initiation and fully required for the maintenance of SUP expression in floral meristems. Their function in control of SUP expression appears to be redundant. Flowers of ap3-3 pi-1 are indistinguishable from ap3-3 and pi-1 flowers, forming both third and fourth whorl organs. This is in agreement with the functioning of AP3 and PI proteins as a DNA binding heterodimer (Riechmann et al., 1996). The amount of SUP mRNA is strongly reduced but yet detectable in ap3-3 pi-1 as in ap3-3 or pi-1. Again, this shows that the initial SUP expression is positively regulated by factors aside from these homeotic genes.
On the other hand, the class $C$ homeotic gene $A G$ functions in a different way. In the strong ag mutant, SUP expression is unaffected in stage 4 but is not detectable later in the whorl 3 organ primordia. This shows that the role of $A G$ is to maintain SUP expression in the floral meristem rather than to control initial expression. Phenotypically, ag and sup mutations exhibit an additive interaction, producing flowers with an indeterminate number of petals interior to whorl 2 in the double mutant (Schultz et al., 1991; Bowman et al., 1992). In ag flowers, despite the lack of SUP expression at stage 5 and later, the structure of whorl 3 is well maintained and $A P 3$ is not ectopically expressed in the interior to whorl 3 (Bowman et al., 1991; Jack et al., 1992). This shows that the very brief SUP expression in ag stage 4 flowers is sufficient to fulfill SUP function in whorl boundary maintenance.

The requirement of $A P 3, P I$, and $A G$ for SUP expression in the maintenance phase is absolute. SUP expression is not maintained when any of these genes loses function. Apparently these homeotic genes coordinate together such that each gene is indispensable for the activation of SUP (see Figure $6 \mathrm{~B}$ ). The role of $L F Y$ in this phase appears to be minimal because SUP expression is maintained in stage 5/6 Ify flowers when AP3 and $P I$ are overexpressed (35S::AP3 35S::PI lfy-6).

\section{Role of as yet Unidentified Genes in SUP Expression}

The requirement of other factors for early SUP expression is further demonstrated by expression analyses in transgenic flowers ectopically expressing homeotic genes. In flowers constitutively expressing $A P 3$ and $P I, S U P$ is not activated in the whorl 4 region of the stage 4 floral meristem, where these homeotic genes as well as $A G$ are expressed. Moreover, constitutive expression of $A P 3, P l$, and $A G$ does not induce SUP expression in whorls 1 and 2, although the transgenic plants form stamens in all floral whorls. The genetic activity required for stamen identity is thus not sufficient to induce SUP expression ectopically in floral meristems. These results again show the requirement of an additional whorl-specific factor to activate SUP expression in whorl 3 or to prevent SUP expression in whorls 1 and 2 as well as in the center of the flower (see Figure 6).

As seen in 35S::AP3 35S::PI Ify-6 flowers, ectopic expression of $A P 3$ and $P I$ partly restores SUP expression in the third whorl region of floral meristem, even in the lfy mutant background. This suggests that the whorl-specific factor delimiting SUP expression to whorl 3 is still active in the absence of $L F Y$ function. Because no other known mutants of floral genes, including genes controlling floral meristem identity (AP1 and UFO), affect early SUP expression, the third whorl-specific pathway appears to be mediated by at least one as yet unidentified gene. Such whorl-specific factors might not necessarily be flower-specific factors, as was proposed for factors interacting with LFY (Parcy et al., 1998). 


\section{Transient Requirement of SUP in Whorl Boundary Maintenance}

As shown by SUP activity in the ag mutant, the temporal window of the SUP requirement appears to be very brief. This is further demonstrated by the interaction between sup and class B homeotic mutations. In the strong mutants ap3-3 and pi-1, early SUP expression is detected only in stage 4 flowers and at a reduced amount. However, the flowers of the double mutants sup ap3 and sup pi are not identical to those of ap3 and pi single mutants. In ap3-3 and pi-1, sepals are formed in two outer whorls, whereas carpels and often filamentous organs are formed in two inner whorls. The structure of the two inner whorls is distinguishable in these flowers by the pattern of emerging organ primordia, especially in early forming flowers. On the other hand, sup ap3-3 and sup pi-1 double mutants produce flowers with one central gynoecium interior to whorl 2. Based on its morphology and its ontogeny (developing from more than two primordia), the gynoecium appears to be formed from whorl 3 carpel primordia, and the formation of whorl 4 is arrested. The further development of the double mutant gynoecium shows characteristics seen in sup mutants: proliferating extra tissues inside of whorl 3 that occasionally form an extra whorl structure, yet undeveloped meristematic structures present at the floral center. These phenotypic correlations reveal that SUP is functional in ap3 and pi mutants. Because early SUP expression is detectable only at stage 4 in ap3 and pi mutants, SUP appears to be required for a short period of time during flower development (possibly $<12 \mathrm{hr}$ ).

\section{Possible SUP Function}

Gradual alteration of $A P 3$ expression in sup mutant flowers suggests two possible functions for SUP in floral meristems (Sakai et al., 1995). In one model, SUP regulates AP3 expression by inhibiting the possible spread of the capability to induce $A P 3$ mRNA from the third whorl into the fourth whorl region. Alternatively, SUP could control the balance of cell proliferation activity in the third and fourth whorl region of the floral meristem. In the latter model, progressive ectopic expression of the class $B$ homeotic genes in the whorl 4 region of sup flowers is the consequence of overproliferation of the third whorl cells and of reduced cell division in fourth whorl cells. The results presented here support the latter model. A nonepistatic relationship between ap3-3/pi-1 and sup-1 demonstrates that SUP function is not simply to control the activity of $A P 3$ and $P I$. The cell division model is also consistent with the phenotype of 35S::AP3 35S::PI sup-1. Flowers of these transgenic plants constitutively expressing $A P 3$ and $P I$ in the sup mutant background exhibit a more enhanced indeterminate fate of floral meristems than do the flowers of 35S::AP3 35S::PI in the wild-type background (Krizek and Meyerowitz, 1996). One plausible role of SUP is therefore to coordinate proliferation of stamen- and carpel- specific meristematic cells, keeping the proper structure of whorls and maintaining the boundary between whorls 3 and 4 at the right position. Because it is expressed in the inner part of whorl 3 but not in whorl 4, SUP appears to control the cell division activity of whorl 4 nonautonomously. SUP also might induce a cell-cell communication mechanism such as that mediated by CLAVATA1 and CLAVATA3 (Fletcher et al., 1999), which allows whorl 3 and 4 cells to receive information from the adjacent floral region. Given that SUP is required for a very short time before the cells in floral whorls undergo extensive divisions to produce organ primordia, possibly the role of SUP is to trigger a cascade of genetic actions that maintain balanced proliferation of two adjacent floral whorls for a long developmental period.

\section{METHODS}

\section{Plant Material}

All plants were grown as described previously (Bowman et al., 1989). To obtain the ap3-3 pi-1 double mutants, homozygous pi-1 flowers were pollinated by pollen from heterozygous ap3-3 mutants. $F_{2}$ plants from the selfed $F_{1}$ were genotyped by polymerase chain reaction (PCR) with the primer sets 5'-AGAGGATAGAGAACCAGACAAATCGA-3'/ 5'-GTTTAGAGAGATGGTGTACGTGG-3' for ap3-3 (Sablowski and Meyerowitz, 1998), and 5'-GATTACTGTTGTCCTTCCATGG-3'/5'-ATCTAGGGTTAAAGATTCAAGGG-3' for pi-1 (Riechmann and Meyerowitz, 1997).

\section{In Situ Hybridization}

Flowers were fixed, embedded, sectioned, hybridized with the ${ }^{35} \mathrm{~S}$ labeled SUP antisense probe, and exposed for 8 to 10 weeks as described previously (Sakai et al., 1995).

The experiments were repeated at least three times for each mutant background.

\section{Scanning Electron and Bright-Field Microscopy}

Flowers were fixed, dried, dissected, and coated for scanning electron microscopy as described previously (Bowman et al., 1989). For histological analyses, flowers were fixed and embedded in Paraplast as they were for in situ hybridization. Floral tissues were sectioned in 6 - or 4- $\mu \mathrm{m}$-thick sections and stained with toluidine blue. The sections were analyzed with a Nikon Eclipse-800 microscope, and the images were captured through the charge-coupled device camera system and processed with the Adobe Photoshop 5.0 program (Adobe Systems, Inc., San Jose, CA).

\section{Strain Construction}

The transgenic line 35S::AP3 was derived from Jack et al. (1994), and 35S::PI was derived from Krizek and Meyerowitz (1996). The AG open reading frame corresponding to ATG-1 (Mizukami and Ma, 1992) was amplified by PCR and cloned into PCGN1547 containing a 
$35 S$ promoter and a $3^{\prime}$-nos sequence (Krizek and Meyerowitz, 1996). The construct was transformed into ecotype No-0 by standard root method (Valvekens et al., 1988) and using the Agrobacterium ASE strain to generate the $35 \mathrm{~S}:: \mathrm{AG}$ line. The 35S::AP3 35S::PI double mutant was constructed by the crossing described by Krizek and Meyerowitz (1996). The triple transgenic line (35S::AP3 35S::PI 35S::AG) was constructed by fertilizing 35S::AG carpels with pollen from 35S::AP3 $35 \mathrm{~S}:: \mathrm{PI}$. Triple transgenics were selected in $\mathrm{F}_{1}$ plants based on their phenotype.

\section{ACKNOWLEDGMENTS}

We thank Catherine Baker, Chiou-Fen Chung, Toshiro Ito, Carolyn Ohno, Doris Wagner, and Eva Ziegelhofer for comments on the manuscript and Pat Koen for assistance with the scanning electron microscope. This work was supported by National Science Foundation Grant No. MCB-9603821 to E.M.M.

Received March 10, 2000; accepted July 24, 2000.

\section{REFERENCES}

Bowman, J.L., Smyth, D.R., and Meyerowitz, E.M. (1989). Genes directing flower development in Arabidopsis. Plant Cell 1, 37-52.

Bowman, J.L., Smyth, D.R., and Meyerowitz, E.M. (1991). Genetic interactions among floral homeotic genes of Arabidopsis. Development 112, 1-20.

Bowman, J.L., Sakai, H., Jack, T., Weigel, D., Mayer, U., and Meyerowitz, E.M. (1992). SUPERMAN, a regulator of floral homeotic genes in Arabidopsis. Development 114, 599-615.

Bowman, J.L., Alvarez, J., Weigel, D., Meyerowitz, E.M., and Smyth, D.R. (1993). Control of flower development in Arabidopsis thaliana by APETALA1 and interacting genes. Development 119, 721-743.

Busch, M.A., Bomblies, K., and Weigel, D. (1999). Activation of a floral homeotic gene in Arabidopsis. Science 285, 585-587.

Fletcher, J.C., Brand, U., Running, M.P., Simon, R., and Meyerowitz, E.M. (1999). Signaling of cell fate decisions by CLAVATA3 in Arabidopsis shoot meristems. Science 283, 1911-1914.

Gaiser, J.C., Robinson-Beers, K., and Gasser, C.S. (1995). The Arabidopsis SUPERMAN gene mediates asymmetric growth of the outer integument of ovules. Plant Cell 7, 333-345.

Goto, K., and Meyerowitz, E.M. (1994). Function and regulation of the Arabidopsis floral homeotic gene PISTILLATA. Genes Dev. 8, 1548-1560.

Huala, E., and Sussex, I.M. (1992). LEAFY interacts with floral homeotic genes to regulate Arabidopsis floral development. Plant Cell 4, 901-913.

Ingram, G.C., Goodrich, J., Wilkinson, M.D., Simon, R., Haughn, G.W., and Coen, E.S. (1995). Parallels between UNUSUAL FLORAL ORGANS and FIMBRIATA, genes controlling flower development in Arabidopsis and Antirrhinum. Plant Cell 7, 1501-1510.

Irish, V.F., and Sussex, I.M. (1990). Function of the apetala-1 gene during Arabidopsis floral development. Plant Cell 2, 741-753.
Jack, T., Brockman, L.L., and Meyerowitz, E.M. (1992). The homeotic gene APETALA3 of Arabidopsis thaliana encodes a MADS box and is expressed in petals and stamens. Cell 68, 683-697.

Jack, T., Fox, G.L., and Meyerowitz, E.M. (1994). Arabidopsis homeotic gene APETALA3 ectopic expression: Transcriptional and posttranscriptional regulation determine floral organ identity. Cell 76, 703-716.

Krizek, B.A., and Meyerowitz, E.M. (1996). The Arabidopsis homeotic genes APETALA3 and PISTILLATA are sufficient to provide the $B$ class organ identity function. Development 122, 11-22.

Levin, J.Z., and Meyerowitz, E.M. (1995). UFO: An Arabidopsis gene involved in both floral meristem and floral organ development. Plant Cell 7, 529-548.

Mandel, M.A., Gustafson-Brown, C., Savidge, B., and Yanofsky, M.F. (1992). Molecular characterization of the Arabidopsis floral homeotic gene APETALA1. Nature 360, 273-277.

Mizukami, Y., and Ma, H. (1992). Ectopic expression of the floral homeotic gene AGAMOUS in transgenic Arabidopsis plants alters floral organ identity. Cell 71, 119-131.

Parcy, F., Nilsson, O., Lee, I., and Weigel, D. (1998). A genetic framework for floral patterning. Nature 395, 561-566.

Riechmann, J.L., and Meyerowitz, E.M. (1997). Determination of floral organ identity by Arabidopsis MADS box domain homeotic proteins AP1, AP3, PI, and AG is independent of their DNA-binding specificity. Mol. Biol. Cell 8, 1243-1256.

Riechmann, J.L., Krizek, B.A., and Meyerowitz, E.M. (1996). Dimerization specificity of Arabidopsis MADS domain homeotic proteins, APETALA1, APETALA3, PISTILLATA, and AGAMOUS. Proc. Natl. Acad. Sci. USA 93, 4793-4798.

Sablowski, R.W.M., and Meyerowitz, E.M. (1998). A homolog of NO APICAL MERISTEM is an immediate target of the floral homeotic genes APETALA3/PISTILLATA. Cell 92, 93-103.

Sakai, H., Medrano, L.J., and Meyerowitz, E.M. (1995). Role of SUPERMAN in maintaining Arabidopsis floral whorl boundaries. Nature 378, 199-203.

Schultz, E.A., Pickett, F.B., and Haughn, G.W. (1991). The FLO10 gene product regulates the expression domain of homeotic genes $A P 3$ and $P I$ in Arabidopsis flowers. Plant Cell 3, 1221-1237.

Smyth, D.R., Bowman, J.L., and Meyerowitz, E.M. (1990). Early flower development in Arabidopsis. Plant Cell 2, 755-767.

Valvekens, D., van Montagu, M., and van Lijsebettens, M. (1988). Agrobacterium tumefaciens-mediated transformation of Arabidopsis thaliana root explants by using kanamycin selection. Proc. Natl. Acad. Sci. USA 85, 5536-5540.

Weigel, D., and Meyerowitz, E.M. (1993). Activation of floral homeotic genes in Arabidopsis. Science 261, 1723-1726.

Weigel, D., Alvarez, J., Smyth, D.R., Yanofsky, M.F., and Meyerowitz, E.M. (1992). LEAFY controls floral meristem identity in Arabidopsis. Cell 69, 843-859.

Wilkinson, M.D., and Haughn, G.W. (1995). UNUSUAL FLORAL ORGANS controls meristem identity and organ primordia fate in Arabidopsis. Plant Cell 7, 1485-1499.

Yanofsky, M.F., Ma, H., Bowman, J.L., Drews, G.N., Feldmann, K.A., and Meyerowitz, E.M. (1990). The protein encoded by the Arabidopsis homeotic gene AGAMOUS resembles transcription factors. Nature 346, 35-39. 